\title{
Integral representations for some weighted classes of functions holomorphic in matrix domains
}

\author{
by M. M. Djrbashian and A. H. Karapetyan (Yerevan)
}

Abstract. In 1945 the first author introduced the classes $H^{p}(\alpha), 1 \leq p<\infty, \alpha>-1$, of holomorphic functions in the unit disk $\mathbb{D}$ with finite integral

$$
\iint_{\mathbb{D}}|f(\zeta)|^{p}\left(1-|\zeta|^{2}\right)^{\alpha} d \xi d \eta<\infty \quad(\zeta=\xi+i \eta)
$$

and established the following integral formula for $f \in H^{p}(\alpha)$ :

$$
f(z)=\frac{\alpha+1}{\pi} \iint_{\mathbb{D}} f(\zeta) \frac{\left(1-|\zeta|^{2}\right)^{\alpha}}{(1-z \bar{\zeta})^{2+\alpha}} d \xi d \eta, \quad z \in \mathbb{D} .
$$

We have established that the analogues of the integral representation (2) hold for holomorphic functions in $\Omega$ from the classes $L^{p}\left(\Omega ;[K(w)]^{\alpha} d m(w)\right)$, where:

1) $\Omega=\left\{w=\left(w_{1}, \ldots, w_{n}\right) \in \mathbb{C}^{n}: \operatorname{Im} w_{1}>\sum_{k=2}^{n}\left|w_{k}\right|^{2}\right\}, K(w)=\operatorname{Im} w_{1}-$ $\sum_{k=2}^{n}\left|w_{k}\right|^{2}$

2) $\Omega$ is the matrix domain consisting of those complex $m \times n$ matrices $W$ for which $I^{(m)}-W \cdot W^{*}$ is positive-definite, and $K(W)=\operatorname{det}\left[I^{(m)}-W \cdot W^{*}\right]$;

3) $\Omega$ is the matrix domain consisting of those complex $n \times n$ matrices $W$ for which $\operatorname{Im} W=(2 i)^{-1}\left(W-W^{*}\right)$ is positive-definite, and $K(W)=\operatorname{det}[\operatorname{Im} W]$.

Here $d m$ is Lebesgue measure in the corresponding domain, $I^{(m)}$ denotes the unit $m \times m$ matrix and $W^{*}$ is the Hermitian conjugate of the matrix $W$.

1. In his 1945 and 1948 papers [4, 5] one of the authors of the present report introduced the classes $H^{p}(\alpha)(1 \leq p<\infty,-1<\alpha<\infty)$ of functions $f(z)$, holomorphic in the unit disk $\mathbb{D}=\{z:|z|<1\}$, for which the integral

$$
\iint_{\mathbb{D}}|f(\zeta)|^{p}\left(1-|\zeta|^{2}\right)^{\alpha} d \xi d \eta \quad(\zeta=\xi+i \eta)
$$

1991 Mathematics Subject Classification: 30E20, 32A07, 32A10, 32A25, 32A35, $32 \mathrm{M} 15$.

Key words and phrases: Siegel domain, matrix domains, generalized unit disk, generalized upper half-plane, weighted classes of holomorphic functions, integral representations. 
is finite $\left(^{1}\right)$. The following theorem was also established.

Theorem A. Any function $f \in H^{p}(\alpha)(1 \leq p<\infty,-1<\alpha<\infty)$ admits the integral representation

$$
f(z)=\frac{1+\alpha}{\pi} \iint_{\mathbb{D}} \frac{f(\zeta)\left(1-|\zeta|^{2}\right)^{\alpha}}{(1-z \bar{\zeta})^{2+\alpha}} d \xi d \eta, \quad z \in \mathbb{D} .
$$

This basic result gave rise to a series of papers devoted to integral and factorization representations of large classes of meromorphic functions. It also found essential application in the solution of several problems of complex analysis. For detailed information we refer the reader to the surveys [6] (1987), [7] (1988) of the first-named author and to the 1988 monograph [3] of A. E. Djrbashyan and F. A. Shamoyan.

As the importance of the classes $H^{p}(\alpha)$ and their integral representations was revealed, investigations devoted to the establishment of analogues of Theorem A for various one-dimensional or multidimensional complex domains became more frequent.

First of all, the known 1958 monograph [14] by L. K. Hua should be mentioned. There integral representations were obtained for holomorphic functions which belong to $L^{2}$-spaces (without weight) in classical matrix domains (Cartan domains). To formulate one of Hua's main results, it is necessary to introduce first some notations.

For any integers $m, n \geq 1$ we shall denote by $M_{m, n}$ the set of all complex $m \times n$ matrices. If $\zeta \in M_{m, n}$, then $\zeta^{*} \in M_{n, m}$ will be the Hermitian conjugate of $\zeta$. We shall denote by $I^{(m)}$ the square $m \times m$ unit matrix. We introduce the Lebesgue measure in $M_{m, n}$ as usual:

$$
d \mu_{m, n}(\zeta)=\prod_{\substack{1 \leq i \leq m \\ 1 \leq j \leq n}} d m_{2}\left(\zeta_{i j}\right),
$$

here $\zeta=\left(\zeta_{i j}\right)_{1 \leq i \leq m, 1 \leq j \leq n} \in M_{m, n}$ and $m_{2}\left(\zeta_{i j}\right)$ is the Lebesgue measure in the plane of the complex variable $\zeta_{i j}$. Note that $M_{1, n}$ coincides with the ordinary complex coordinate space $\mathbb{C}^{n}$ and $\mu_{1, n}$ is the $2 n$-dimensional Lebesgue measure in $\mathbb{C}^{n} \cong \mathbb{R}^{2 n}$.

The domain

$$
\text { (4) } \quad \mathcal{R}_{m, n}=\left\{\zeta \in M_{m . n}: I^{(m)}-\zeta \zeta^{*} \text { is strictly positive-definite }\right\}
$$

is called the generalized unit disk in $M_{m, n}$. It is easy to see that $\mathcal{R}_{1, n}$ coincides with the unit ball $\mathbb{B}_{n}=\left\{\xi=\left(\zeta_{1}, \ldots, \zeta_{n}\right) \in \mathbb{C}^{n}:|\zeta|^{2}=\sum_{k=1}^{n}\left|\zeta_{k}\right|^{2}<1\right\}$ in $\mathbb{C}^{n}=M_{1, n}$.

$\left({ }^{1}\right)$ By reasons not quite clear to us the name "weighted Bergman spaces" established itself for these classes in recent years. It does not, evidently, reflect the real essence of the matter. 
The result of L. K. Hua is

TheOREM B. Any holomorphic function $f \in L^{2}\left(\mathcal{R}_{m, n} ; d \mu_{m, n}\right)$ admits the integral representation

$$
f(Z)=c_{m, n} \int_{\mathcal{R}_{m, n}} \frac{f(\zeta)}{\left[\operatorname{det}\left(I^{(m)}-Z \zeta^{*}\right)\right]^{m+n}} d \mu_{m, n}(\zeta), \quad Z \in \mathcal{R}_{m, n},
$$

where

$$
c_{m, n}=\frac{\prod_{l=1}^{m+n} \Gamma(l)}{\prod_{k=1}^{m} \Gamma(k) \prod_{j=1}^{n} \Gamma(j)} \cdot \frac{1}{\pi^{m n}} .
$$

Since $\mathcal{R}_{m, n}$ coincides with the unit disk $\mathbb{D} \subset \mathbb{C}$ for $m=n=1$, Theorem $\mathrm{B}$ is a multidimensional analogue of Theorem A but, of course, only for the particular values $p=2, \alpha=0$ of the parameters. Moreover, note that when $m=1, n \geq 1$, Theorem $\mathrm{B}$ establishes the integral representation of holomorphic functions which belong to the space $L^{2}$ (without weight again) in the unit ball $\mathbb{B}_{n} \subset \mathbb{C}^{n}$.

Thus, Theorem B did not answer the question whether there existed multidimensional analogues of the representation (2) for holomorphic functions from weighted $L^{p}$-spaces, for $p \in[1, \infty)$. However, later investigations gave the solution of this problem for certain domains. More precisely, the following theorem turned out to be true.

Theorem C. Let $1 \leq p<\infty,-1<\alpha<\infty$ and let the complex number $\beta$ satisfy

$$
\begin{array}{ll}
\operatorname{Re} \beta>\frac{1+\alpha}{p}-1 & \text { if } 1<p<+\infty, \\
\operatorname{Re} \beta \geq \alpha & \text { if } p=1 .
\end{array}
$$

Then any holomorphic function $f$ in $\mathbb{B}_{n}$ for which

$$
\int_{\mathbb{B}_{n}}|f(\zeta)|^{p}\left(1-|\zeta|^{2}\right)^{\alpha} d \mu_{1, n}(\zeta)<\infty
$$

admits the integral representation

$$
f(z)=\frac{\Gamma(1+n+\beta)}{\Gamma(1+\beta) \pi^{n}} \int_{\mathbb{B}_{n}} \frac{f(\zeta)\left(1-|\zeta|^{2}\right)^{\beta}}{\left(1-z \zeta^{*}\right)^{1+n+\beta}} d \mu_{1, n}(\zeta), \quad z \in \mathbb{B}_{n}
$$

For $n=1$, i.e. in the case of the unit disk $\mathbb{D} \subset \mathbb{C}$, this easily follows from Theorem A, which, as has already been mentioned, was established in the 1940s by one of the present authors in [4, 5]. For $n>1$, but $\alpha=0$, Theorem C was first established by F. Forelli and W. Rudin in their 1974 paper [12], where, however, the case $p=1, \operatorname{Re} \beta=0$ failed to be considered. In the 1988 survey [7] it is shown that even in the general case, i.e. for $n>1$ and $-1<\alpha<\infty$, Theorem $\mathrm{C}$ can be established by the methods 
already developed in the forties, when Theorem A was proved $\left(^{2}\right)$. Note, by the way, that the statement of Theorem $\mathrm{C}$ essentially generalizes that of theorem B only for $m=1, n \geq 1$. It should also be mentioned that in M. Stool's 1977 paper [15] the analogue of the integral representation (9) was established for arbitrary bounded symmetric domains including the domains $\mathcal{R}_{m, n}(m, n \geq 1)$. But all that was done for spaces without weight, just as in the paper of F. Forelli and W. Rudin [12]. Finally in 1978 S. A. Dautov and G. M. Henkin [2] obtained the analogues of (9) for smooth functions in strictly pseudoconvex domains which are much more general than the unit ball $\mathbb{B}_{n}$.

The result we established in 1989 (see [10]) is a further generalization of Theorems A, B, C and may be formulated as follows.

TheOREM 1. Let $1 \leq p<\infty,-1<\alpha<\infty$ and let the complex number $\beta$ satisfy

$$
\begin{array}{ll}
\operatorname{Re} \beta>\frac{1+\alpha}{p}-1 & \text { if } 1<p<+\infty, \\
\operatorname{Re} \beta \geq \alpha & \text { if } p=1 .
\end{array}
$$

Then any holomorphic function $f$ in $\mathcal{R}_{m, n}(m, n \geq 1)$ for which

$$
\int_{\mathcal{R}_{m, n}}|f(\zeta)|^{p}\left[\operatorname{det}\left(I^{(m)}-\zeta \zeta^{*}\right)\right]^{\alpha} d \mu_{m, n}(\zeta)<\infty
$$

admits the integral representation

$$
\begin{aligned}
& f(Z)=c_{m, n}(\beta) \int_{\mathcal{R}_{m, n}} \frac{f(\zeta)\left[\operatorname{det}\left(I^{(m)}-\zeta \zeta^{*}\right)\right]^{\beta}}{\left[\operatorname{det}\left(I^{(m)}-Z \zeta^{*}\right)\right]^{m+n+\beta}} d \mu_{m, n}(\zeta) \\
& \\
& Z \in \mathcal{R}_{m, n}
\end{aligned}
$$

where

$$
c_{m, n}(\beta)=\frac{\prod_{l=1}^{m+n} \Gamma(l+\beta)}{\prod_{k=1}^{m} \Gamma(k+\beta) \prod_{j=1}^{n} \Gamma(j+\beta)} \cdot \frac{1}{\pi^{m n}} .
$$

We see that Theorem 1 is an extension of Theorem $\mathrm{C}$ and precisely coincides with it for $m=1, n \geq 1$. On the other hand, Theorem 1 is an essential generalization of Theorem $\mathrm{B}$ and coincides with it for special values of parameters: $p=2$ and $\alpha=\beta=0$. We think that the methods developed in Hua's monograph [14] for the proof of Theorem B cannot be applied in the more general case of Theorem 1 .

2. So far we have considered analogues or generalizations of the integral representation (2) for (multidimensional) bounded domains. But there also

$\left({ }^{2}\right)$ The parameter $\beta$ of (7) was supposed to be real in the survey [7], but the proof presented there obviously remains valid also for complex values of $\beta$. 
exist investigations where analogues of (2) are obtained for certain classes of holomorphic functions in unbounded domains. In this connection we mention the 1964 paper of S. G. Gindikin [13] devoted to analysis in Siegel domains. These domains are defined as follows:

$$
\begin{aligned}
D=\left\{\eta=(z, u) \in \mathbb{C}^{n+m}, z=x+i y \in \mathbb{C}^{n}, u\right. & \in \mathbb{C}^{m}: \\
& y-F(u, u) \in V\},
\end{aligned}
$$

where $V \subset \mathbb{R}^{n}(n \geq 1)$ is a sharp open convex cone and the mapping $F: \mathbb{C}^{m} \times \mathbb{C}^{m} \rightarrow \mathbb{C}^{n}(m \geq 0)$ has some natural properties, usually referred to by means of the term " $V$-Hermitian form". By the way, note that $D$ is a tube domain in $\mathbb{C}^{n}$ over the cone $V \subset \mathbb{R}^{n}$ if $m=0$, i.e. if there is not any form $F$. In particular, S. G. Gindikin constructed the reproducing kernels (in the form of Laplace integrals) for holomorphic functions in unweighted $L^{2}$ spaces in Siegel domains. Moreover, he wrote down the explicit form of these kernels for the so-called affine-homogeneous Siegel domains. Later (1980), R. Coifman and R. Rochberg [1] indicated that Gindikin's methods allow one to obtain the explicit form of the reproducing kernels for some weighted $L^{2}$-spaces of holomorphic functions in symmetric affine-homogeneous Siegel domains.

In any case, there remained open the question of existence of integral representations for holomorphic functions in weighted $L^{p}$-spaces in unbounded domains, for $1 \leq p<\infty$. In other words, the problem of obtaining the most complete analogues of Theorem A for some (even the simplest) unbounded domains remained unsolved.

In 1985 M. M. Djrbashian and A. ̇̀. Djrbashyan [8] proved the following theorem by applying the integral representation (2) and a special passage to the limit.

TheOREM D. Let $1 \leq p<\infty$ and $-1<\alpha<\infty$. Then any holomorphic function $f$ in the upper half-plane $\Pi_{+}=\{\omega \in \mathbb{C}: \operatorname{Im} \omega>0\}$ with

$$
\iint_{\Pi_{+}}|f(\omega)|^{p}(\operatorname{Im} \omega)^{\alpha} d u d v<\infty \quad(\omega=u+i v)
$$

admits the integral representation

$$
f(w)=\frac{2^{\alpha}(\alpha+1)}{\pi} \iint_{\Pi_{+}} \frac{f(\omega)(\operatorname{Im} \omega)^{\alpha}}{[i(\bar{\omega}-w)]^{2+\alpha}} d u d v, \quad w \in \Pi_{+} .
$$

In the same paper the corresponding projection theorem was also obtained.

Making use of a similar limit passage and basing on the integral representation in the unit ball $\mathbb{B}_{n} \subset \mathbb{C}^{n}$ (see Theorem $\mathrm{C}$ ) we have established an 
analogue of Theorem D for a Siegel type domain

$$
\Omega_{n}=\left\{\omega=\left(\omega_{1}, \ldots, \omega_{n}\right) \in \mathbb{C}^{n}: \operatorname{Im} \omega_{1}>\sum_{k=2}^{n}\left|\omega_{k}\right|^{2}\right\} .
$$

This domain is biholomorphically equivalent to the unit ball $\mathbb{B}_{n}$ and coincides with the upper half-plane $\Pi_{+} \subset \mathbb{C}$ for $n=1$. More precisely, the following 1987 theorem [9] turns out to be valid.

TheOREm 2. Let $1 \leq p<\infty,-1<\alpha<\infty$ and let the complex number $\beta$ satisfy

$$
\begin{array}{ll}
\operatorname{Re} \beta>\frac{1+\alpha}{p}-1 & \text { if } 1<p<\infty, \\
\operatorname{Re} \beta \geq \alpha & \text { if } p=1 .
\end{array}
$$

Then any holomorphic function $f$ in $\Omega_{n}$ with

$$
\|f\|_{p, \alpha}^{p} \equiv \int_{\Omega_{n}}|f(\omega)|^{p}\left[\operatorname{Im} \omega_{1}-\sum_{k=2}^{n}\left|\omega_{k}\right|^{2}\right]^{\alpha} d \mu_{1, n}(\omega)<\infty
$$

admits the integral representation

(18) $f(w)=2^{n-1+\beta} c_{1, n}(\beta) \int_{\Omega_{n}} \frac{f(\omega)\left[\operatorname{Im} \omega_{1}-\sum_{k=2}^{n}\left|\omega_{k}\right|^{2}\right]^{\beta} d \mu_{1, n}(\omega)}{\left[i\left(\bar{\omega}_{1}-w_{1}\right)-2 \sum_{k=2}^{n} w_{k} \bar{\omega}_{k}\right]^{1+n+\beta}}$,

$$
w \in \Omega_{n} .
$$

Moreover, under the condition $\operatorname{Re} \beta>(1+\alpha) / p-1(1 \leq p<\infty)$ the integral operator generated in a natural way by the representation (18) is a bounded projection from the function space $\left\{f:\|f\|_{p, \alpha}<\infty\right\}$ onto the subspace of holomorphic functions.

Later on (1989) we found out [11] that if we make use of the same idea of special limit passage and base upon the integral representation (11) of Theorem 1, then the statement of Theorem $\mathrm{D}$ can be extended to the case of another multidimensional analogue of the upper half-plane, quite different from $\Omega_{n}$. We mean the so-called generalized upper half-planes $\pi_{n}(n \geq 1)$, defined as

$$
\pi_{n}=\left\{\omega \in M_{n, n}: \operatorname{Im} \omega=\left(\omega-\omega^{*}\right) / 2 i \text { is strictly positive-definite }\right\} .
$$

The domain $\pi_{n}$ is biholomorphically equivalent to the generalized unit disk $\mathcal{R}_{n, n}$.

So we have proved

TheOREM 3. Let $n \geq 1,1 \leq p<\infty$ and let $\alpha>\max \{-1 ; p(n-1)-$ $(3 n-1)\}$. Then any holomorphic function $f$ in $\pi_{n}$ with

$$
\|f\|_{p, \alpha}^{p} \equiv \int_{\pi_{n}}|f(\omega)|^{p}[\operatorname{det}(\operatorname{Im} \omega)]^{\alpha} d \mu_{n, n}(\omega)<\infty
$$


admits the integral representation

$$
\begin{aligned}
& f(w)=2^{n \beta} \cdot c_{n, n}(\beta) \cdot \int_{\pi_{n}} \frac{f(\omega)[\operatorname{det}(\operatorname{Im} \omega)]^{\beta}}{\left[\operatorname{det}\left(i\left(\omega^{*}-w\right)\right)\right]^{2 n+\beta}} d \mu_{n, n}(\omega), \\
& w \in \pi_{n},
\end{aligned}
$$

where

$$
\begin{array}{ll}
\operatorname{Re} \beta>\frac{1+\alpha}{p}-1 & \text { if } 1<p<\infty, \\
\operatorname{Re} \beta \geq \alpha & \text { if } p=1 .
\end{array}
$$

Moreover, the integral operator generated by the right hand side of (21) is a bounded projection from $\left\{f:\|f\|_{p, \alpha}<\infty\right\}$ onto its subspace of holomorphic functions, provided $1 \leq p<\infty, \alpha>p(n-1)-n$ and $\operatorname{Re} \beta>(\alpha+n) / p-1$.

\section{References}

[1] R. R. Coifman and R. Rochberg, Representation theorems for holomorphic and harmonic functions in $L^{p}$, Astérisque 77 (1980), 11-66.

[2] Š. A. Dautov and G. M. Henkin, The zeroes of holomorphic functions of finite order and weight estimates for solutions of the $\bar{\partial}$-equation, Mat. Sb. 107 (1978), 163-174 (in Russian).

[3] A. È. Djrbashyan and F. A. Shamoyan, Topics in the Theory of $A_{\alpha}^{p}$ Spaces, Teubner-Texte zur Math. 105, Teubner, Leipzig 1988.

[4] M. M. Djrbashian, On the representability of certain classes of functions meromorphic in the unit disk, Akad. Nauk Armyan. SSR Dokl. 3 (1945), 3-9 (in Russian).

[5] -, On the problem of representing analytic functions, Soobshch. Inst. Mat. Mekh. Akad. Nauk Armyan. SSR 2 (1948), 3-40 (in Russian).

[6] -, A survey of some achievements of Armenian mathematicians in the theory of integral representations and factorization of analytic functions, Mat. Vesnik 39 (1987), 263-282.

[7] _, A brief survey of the results obtained by Armenian mathematicians in the field of factorization of meromorphic functions and its applications, Izv. Akad. Nauk Armyan. SSR Ser. Mat. 23 (6) (1988), 517-545 (in Russian).

[8] M. M. Djrbashian and A. È. Djrbashyan, Integral representations for some classes of functions analytic in the half-plane, Dokl. Akad. Nauk SSSR 285 (3) (1985), 547-550 (in Russian).

[9] M. M. Djrbashian and A. H. Karapetyan, Integral representations for some classes of functions analytic in a Siegel domain, Izv. Akad. Nauk Armyan. SSR Ser. Mat. 22 (4) (1987), 399-405 (in Russian).

[10] - , - Integral representations in the generalized unit disk, ibid. 24 (6) (1989), 523-546 (in Russian).

[11] - - - Integral representations in the generalized upper half-plane, ibid. 25 (6) (1990) (in Russian).

[12] F. For elli and W. Rudin, Projections on spaces of holomorphic functions in balls, Indiana Univ. Math. J. 24 (6) (1974), 593-602. 
[13] S. G. Gindikin, Analysis in homogeneous domains, Uspekhi Mat. Nauk 19 (4) (1964), 3-92 (in Russian).

[14] L. K. Hua, Harmonic Analysis of Functions of Several Complex Variables in the Classical Domains, Inostr. Liter., Moscow 1959 (in Russian).

[15] M. Stoll, Mean value theorems for harmonic and holomorphic functions on bounded symmetric domains, J. Reine Angew. Math. 290 (1977), 191-198.

Remark. The journal "Izv. Akad. Nauk Armyan. SSR Ser. Mat." is translated into English in the same issues of "Soviet Journal of Contemporary Mathematical Analysis, Armenian Academy of Sciences", Allerton Press, Inc., USA.

INSTITUTE OF MATHEMATICS

ARMENIAN ACADEMY OF SCIENCES

MARSHAL BAGRAMIAN AVE., 24-B

375019 YEREVAN, ARMENIA

U.S.S.R. 